\title{
ANALISE QUALI-QUANTITATIVA DO EPIFITISMO VASCULAR NA ARBORIZAÇÃO DE PARANÁ DO OESTE E SUAS INTERAÇÕES
}

\author{
QUALI-QUANTITATIVE EVALUATION OF VASCULAR EPIPHYTISM IN \\ AFFORESTATION OF PARANÁ DO OESTE AND ITS INTERACTIONS
}

\author{
Jhonny Ângelo Barbieri', Francisco Ferreira Martins Neto², Yara Campos Miranda ${ }^{3}$, Rafael de Oliveira \\ Bailão ${ }^{4}$, João Gabriel da Costa Bertoli5 ${ }^{5}$ Antônio dos Santos Filho ${ }^{6}$, Marcelo Galeazzi Caxambu ${ }^{7}$
}

\begin{abstract}
RESUMO
As epífitas são plantas que utilizam o substrato arbóreo como apoio, sendo consideradas bioindicadoras da qualidade ambiental, podendo modificar sua estrutura e abundância de acordo com a presença de qualquer alteração antrópica. Este trabalho tem como objetivo realizar um censo da arborização viária e analisar a relação entre o epifitismo e as árvores das espécies Ligustrum lucidum (Oleaceae) e Handroanthus heptaphyllus (Bignoniaceae) em Paraná do Oeste, distrito do município de Moreira Sales. As amostras coletadas foram identificadas no Herbário da Universidade Tecnológica Federal do Paraná, Campus Campo Mourão (HCF). Para testar a afinidade das espécies por um determinado tipo de substrato, foi utilizado o método do valor indicador (IndVal). Foram identificados 518 indivíduos arbóreos no município, sendo 10 indivíduos da espécie $H$. heptaphyllus e 219 de L. lucidum representando, respectivamente, 1,9\% e 42,3\%. Encontrou-se também 16 espécies de epífitas, destacando-se as famílias Bromeliaceae, Orchidaceae e Polypodiaceae. Deste total, 38 indivíduos estavam dispostos no substrato H. heptaphyllus e 127 indivíduos no substrato L. Iucidum. O índice de afinidade proposto apontou afinidade de $80 \%$ entre Pleopeltis pleopeltifolia (Polypodiaceae) e o substrato L. lucidum, enquanto Dendrobium nobile (Orchidaceae) apresentou $35 \%$ de afinidade com o substrato $H$. heptaphyllus.
\end{abstract}

Palavras-chave: Exótica invasora; Epífitas vasculares; Bray-Curtis.

\begin{abstract}
Epiphytes are plants that use the arboreal substrate as support, considered as bio-indicators of environmental quality, being able to modify its structure and abundance according to the presence of any anthropic alteration. This study aimed to carry out a census of the urban arborization and analyze the relationship between the epiphytism and the trees species Ligustrum lucidum (Oleaceae) and Handroanthus heptaphyllus (Bignoniaceae) in Paraná do Oeste, district of Moreira Sales. The samples were identified in the Herbarium of the Federal Technological University of Paraná Campus Campo Mourão (HCF). To test the affinity of the species for a particular type of substrate, we used the indicator value method IndVal. A total of 518 trees were identified with $H$. heptaphyllus representing $1.9 \%$ of the total and L. lucidum representing $42.3 \%$. There were also 16 species of epiphytes, highlighting Bromeliaceae, Orchidaceae, and Polypodiaceae families. Of this, 38 individuals were found in the substrate $H$. heptaphyllus and 127 individuals in substrate L. lucidum. The proposed index affinity showed an affinity of $80 \%$ between Pleopeltis pleopeltifolia (Polypodiaceae) and substrate L. lucidum, while Dendrobium nobile (Orchidaceae) showed an affinity of $35 \%$ with the substrate $H$. heptaphyllus.
\end{abstract}

Keywords: Alien Species; Vascular Epiphytes; Bray-Curtis.

Recebido em 22.05.2017 e aceito em 22.09.2017

1 Engenheiro Ambiental. Especialista em Economia Ambiental. Especialização em Projetos Sustentáveis na Universidade Federal do Paraná. Campo Mourão - PR. jhonnyabarbieri@gmail.com

2 Engenheiro Ambiental, Bacharel. Mestrando em Ciências e Tecnologia Ambiental na UFTM. Uberaba - MG. neto.fm@hotmail.com 3 Engenheira Ambiental, Bacharel. Mestranda em Engenharia Urbana na UEM. Maringá - PR, yaracamposmiranda1@gmail.com

4 Engenheiro Ambiental, Bacharel. Mestrando em Bioenergias na UEM. Maringá - PR, rafabailao@hotmail.com

5 Engenheiro Ambiental, Bacharel. Professor na Escola Técnica Dr. Luiz César Couto. Quatá - SP, jg.bertoli@gmail.com

6 Engenheiro Ambiental, Bacharel. UTFPR - Campus Campo Mourão. Campo Mourão - PR, toh itai@hotmail.com

7 Engenheiro Florestal. Dr.,Professor Adjunto na UTFPR - Campus Campo Mourão. Campo Mourão - PR, mcaxambu@utfpr.edu.br 


\section{INTRODUÇÃO}

A arborização urbana é um elemento fundamental para a qualidade de vida das pessoas, podendo ser considerada uma das principais fontes de conservação ambiental da urbe, proporcionando abrigo a fauna e preservando espécies vegetais (OLDFIELD, et al., 2014). Quando associadas a epífitas, otimizam estes benefícios, visto que proporcionam uma maior heterogeneidade vegetal ao ecossistema urbano.

Epífitas são indivíduos que utilizam outras plantas como suporte, pertencendo a várias famílias botânicas, destacando-se Orchidaceae, Araceae, Bromeliaceae, Polypodiaceae, Gesneriaceae, Piperaceae. A ocupação do forófito, comumente denominado árvore hospedeira, pode estar relacionada a diversos fatores abióticos, tais como tipo de substrato para fixação, luz e umidade. (RITTER et al, 2014).

A presença de epífitas ocasionam relevante interação entre avifauna na busca por alimento, proporcionando dispersão de sementes, sendo as espécies das famílias Polypodiaceae e Bromeliaceae típicas de espaços antropizados (BATAGHIN; BARROS; PIRES, 2010). Além disto, beneficiam os hospedeiros com a atração de polinizadores e material para a confecção de ninhos (LAPO; MAGENTA, 2014).

Outro aspecto importante da presença das epífitas nas árvores urbanas, é que de acordo com Bermudez, Rodrigues e Pignata (2009) estas funcionam como bioindicadores de qualidade ambiental, apresentando modificações em sua estrutura, por vezes acumulando partículas de poluentes em seus tecidos ou apresentando lesões em seus aspectos morfológicos de acordo com a presença de poluentes atmosféricos. Gandolfi et al. (2017), ainda afirmam que algumas espécies possuem a capacidade de absorver poluentes, auxiliando na manutenção da qualidade do ar urbano.

Há um crescimento nos estudos sobre epífitas nas últimas décadas, abordando não só a composição florística, mas também a distribuição altitudinal, síndromes de polinização e dispersão, entre outros (DETTKE; ORFRINI; MILANEZE-GUTIERRE, 2008). No estado do Paraná, uma série de estudos vêm sendo realizados, entre estes Geraldino et al. (2010) analisando a flora epifítica em uma área de ecótono de Floresta Estacional Semidecidual com Floresta Ombrófila Mista; Cervi e Borgo (2007) em um estudo qualitativo de espécies de epífitas vasculares encontrados no Parque Nacional do Iguaçu. Os trabalhos que envolvem o epifitismo ocorrente na arborização urbana são raros e recentes, como em Ritter et al. (2014) a partir de um levantamento de epífitas presentes na arborização urbana no município de Farol - PR e Devens et al., (2016) em uma análise de epifitismo na cidade de Luiziana - PR.

Diante de sua importância, este trabalho tem como objetivo realizar o censo da arborização urbana e a presença de epífitas vasculares, bem como analisar a relação existente 
entre o epifitismo e as árvores das espécies Ligustrum lucidum W T. Ailton (Oleaceae) e Handroanthus heptaphyllus (Vell.) Mattos (Bignoniaceae) em Paraná do Oeste, distrito do município de Moreira Sales - PR.

\section{MATERIAL E MÉTODOS}

\section{Caracterização da área de estudo}

A área de estudo (Figura 1) encontra-se no distrito de Paraná do Oeste, pertencente ao município de Moreira Sales, localizado no terceiro planalto paranaense, região Centro Ocidental do Paraná, entre as coordenadas geográficas $24^{\circ} 03^{\prime} 44^{\prime \prime}$ de Latitude Sul e 5300'25" de Longitude Oeste, com altitude média de 450 metros, área de 357,358 km², com população estimada em 12.664 habitantes em 2016 (IBGE, 2016).

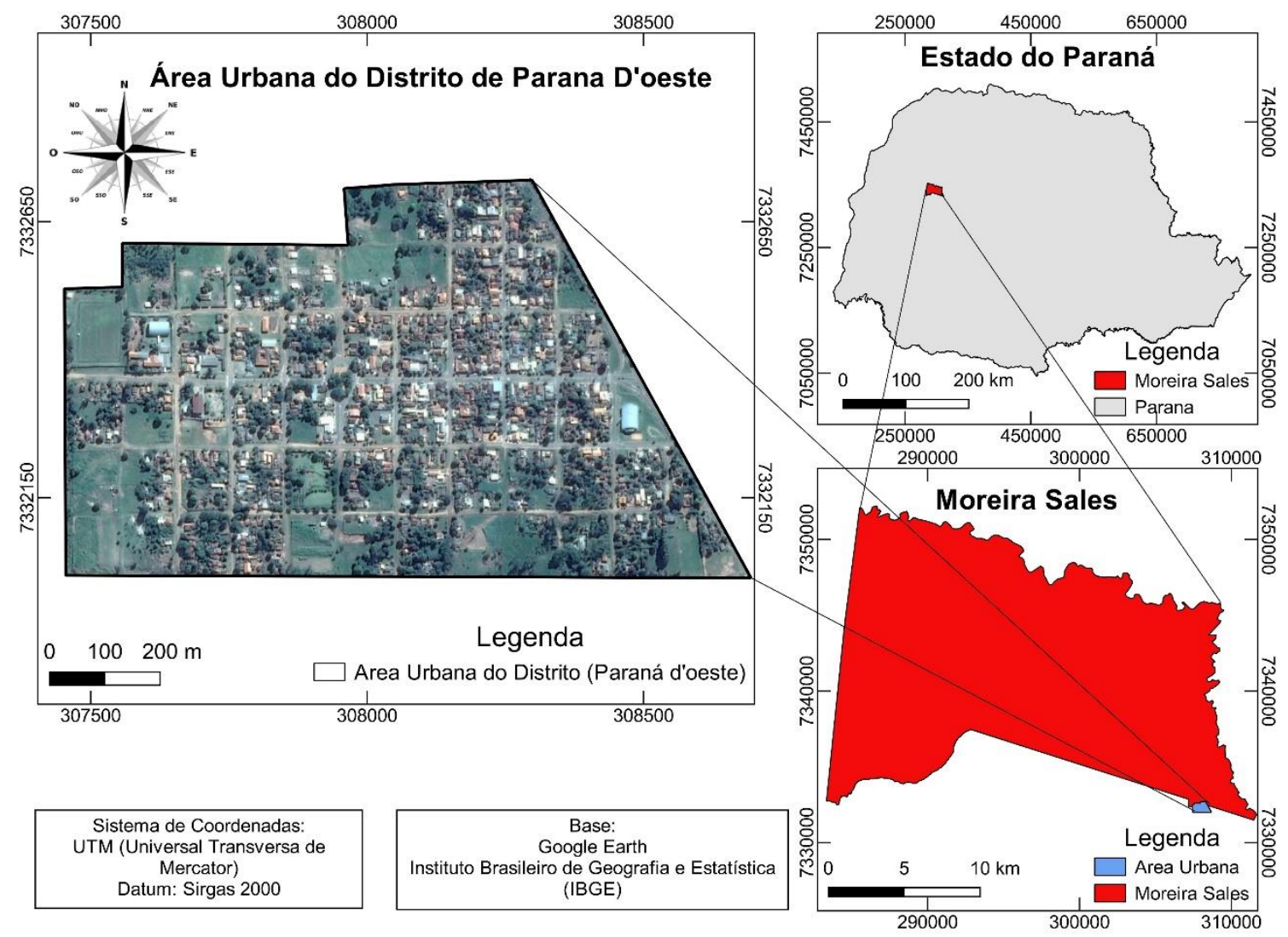

Figura 1. Localização da área urbana do distrito de Paraná D'Oeste - Paraná, Brasil

Figure 1. Location of the urban area of district Paraná D'Oeste - Paraná, Brazil 
A topografia é plana e ligeiramente ondulada e o distrito pertence à bacia hidrográfica do Rio Piquirí (ITCG, 2010). O solo predominante na região de Paraná do Oeste é o Argissolo Vermelho Eutrófico que é descrito como solo medianamente profundo, moderadamente drenado, com horizonte $B$ textural, de cores vermelhas e textura argilosa e com baixos teores de matéria orgânica (EMBRAPA, 2007).

O clima é definido como Cwa e Cfa de acordo com a classificação climática de KoppenGeiger, com a predominância do Cfa subtropical úmido mesotérmico, com verões quentes e geadas pouco frequentes, com tendência de concentração das chuvas nos meses de verão, sem estação seca definida. A média das temperaturas dos meses mais quentes é superior a $22^{\circ} \mathrm{C} \mathrm{e}$ a dos meses mais frios é inferior a $18^{\circ} \mathrm{C}$. Os índices pluviométricos variam de 500 a $600 \mathrm{~mm}$ no trimestre com maior concentração de chuvas (dezembro, janeiro e fevereiro) e 250 a 350mm nos meses de junho, julho e agosto, perfazendo o período seco (IAPAR, 2009).

De acordo com a classificação proposta por Roderjan et al. (2002), a vegetação original do Distrito de Paraná do Oeste é composta predominantemente pela Floresta Estacional Semidecidual Submontana e, nas margens dos rios aparece a Floresta Estacional Semidecidual Aluvial.

\section{Levantamento quali-quantitativo}

Para a coleta dos dados das epífitas foram necessárias duas expedições ao distrito de Paraná do Oeste durante o período de abril a maio de 2013. Na primeira coleta foi conduzido um levantamento florístico total das epífitas da arborização do distrito, por meio de escalada natural do forófito, utilizando uma tesoura de poda para coletar as epífitas. Quando não foi possível realizar identificação in situ, estas foram acondicionadas em sacos plásticos para o armazenamento e transporte ao Herbário da Universidade Tecnológica Federal do Paraná Campus Campo Mourão (HCF) para posterior identificação.

Após coleta e identificação, as espécies arbóreas, Ligustrum lucidum W.T. Aiton (Oleaceae) e Handroanthus heptaphyllus (Vell.) Mattos (Bignoniaceae) foram selecionadas para fins de se verificar se estas apresentavam relação com epifitismo. A escolha destas espécies se deu em função de ambas apresentarem as maiores populações de perenifólias e caducas, respectivamente, na arborização urbana. Foram então selecionados cinco indivíduos de cada espécie para a avaliação.

Os testes estatísticos foram realizados por meio do software Bioestat 5.0. A partir da identificação das epífitas, foram conduzidas análise de cluster, utilizada para avaliar os padrões espaciais das epífitas em dois tratamentos (Handroanthus x Ligustrum), utilizando o método do 
valor indicador (IndVal) que indica possíveis associações entre as espécies e os habitats analisados. Esta análise consiste na combinação da especificidade da espécie ao elemento do habitat em que ocorre $\left(A_{i j}\right)$ e o grau de fidelidade dessa relação $\left(B_{i j}\right)$ (DUFRÊNE, LEGENDRE, 1997), calculados pela seguinte fórmula:

$$
\begin{aligned}
\text { IndVal } & =A_{\mathrm{ij}} \times \mathrm{B}_{\mathrm{ij}} \times 100 \\
\mathrm{~A}_{\mathrm{ij}} & =\frac{\text { Nindiv }_{\mathrm{ij}}}{\text { Nindiv }_{\mathrm{i}}} \\
\mathrm{B}_{\mathrm{ij}} & =\frac{\text { Nlocais }_{\mathrm{ij}}}{\text { Nlocais }_{\mathrm{i}}}
\end{aligned}
$$

Sendo:

$A_{i j}=$ grau da especificidade (medida da exclusividade da espécie em um habitat)

Nindiv $_{\mathrm{ij}}=$ valor médio de indivíduos da espécie i em todos os locais do grupo j

Nindiv $_{\mathrm{i}}=$ somatória da média dos indivíduos da espécie i em todos os grupos

$\mathrm{B}_{\mathrm{ij}}=$ grau de fidelidade (medida de ocorrência dentro do mesmo habitat)

Nlocais $_{\mathrm{ij}}=$ número de locais no grupo j onde a espécie i está presente,

Nlocais $_{\mathrm{i}}=$ número total de locais nesse grupo

A matriz de dados foi transformada em $\log 10(x+1)$ para normalizar suas escalas. Esta análise foi realizada a partir da similaridade de Bray-Curtis, sendo que para a análise de cluster os grupos foram unidos pelo método de agrupamento de médias aritméticas não ponderadas (UPGMA) (ROHLF, 2001).

Para testar a afinidade das espécies por um determinado tipo de substrato (Handroanthus x Ligustrum), foi utilizado o método do valor indicador (IndVal) (DUFENE; LEGENDRE, 1997), o qual relaciona a abundância relativa de espécies e sua frequência relativa em vários grupos. A abundância relativa consiste na razão entre o número de indivíduos de uma espécie em relação ao total de indivíduos (ni/N) e caracteriza o tamanho da população de uma espécie em um determinado habitat. A frequência relativa é determinada pela razão entre a porcentagem de determinada espécie pela somatória de todas e expressa o número de ocorrências desta espécie em relação às demais.

\section{RESULTADOS E DISCUSSÃO}

Em Paraná do Oeste foram registrados 518 indivíduos arbóreos (Tabela 1), sendo que 10 espécies representam $87,8 \%$ da frequência total das árvores encontradas no distrito com ampla predominância de plantas exóticas, totalizando 20 das 29 espécies encontradas. 
Tabela 1. Espécies encontradas na arborização urbana de acompanhamento viário do distrito de Paraná do Oeste, município de Moreira Sales - PR, Brasil. № - número de indivíduos; N - Nativa; NPR - Nativa do Paraná; E - Exótica; El - Exótica e Invasora; F - Frequência relativa de ocorrência

Table 1. Species found in the urban afforestation of roadside in the district of Paraná do Oeste, municipality of Moreira Sales - PR, Brazil. Number of individuals; N - Native; N-PR - native of Paraná State; E - Exotic; El - Exotic and Invasive; F - Relative frequency of occurrence

\begin{tabular}{|c|c|c|c|c|}
\hline Família & Espécie & № & & $F(\%)$ \\
\hline \multirow{2}{*}{ Anacardiaceae } & Mangifera indica L. & 5 & $E$ & 0,965 \\
\hline & Schinus molle $\mathrm{L}$ & 16 & N-PR & 3,089 \\
\hline Araucariaceae & Araucaria columnaris Hook & 1 & $E$ & 0,193 \\
\hline \multirow{2}{*}{ Arecaceae } & Dypsis lutescens (H. Wendl.) Beentje\&J. Dransf. & 2 & $\mathrm{E}$ & 0,386 \\
\hline & Livistona chinensis $\mathrm{R} . \mathrm{Br}$. & 20 & $E$ & 3,861 \\
\hline \multirow{3}{*}{ Bignoniaceae } & Handroanthus chrysotrichus (Mart. ex A. DC.) Mattos & 1 & $\mathrm{~N}$ & 0,193 \\
\hline & Handroantus heptaphyllus (Vell.) Mattos & 10 & N-PR & 1,931 \\
\hline & Spathodea nilotica Seem & 5 & El & 0,965 \\
\hline Boraginaceae & Cordia abyssinica R.Br. & 5 & $E$ & 0,965 \\
\hline Chrysobalanaceae & Licania tomentosa (Benth) Fritsh & 16 & $\mathrm{~N}$ & 3,089 \\
\hline Combretaceae & Terminalia catappa L. & 5 & $\mathrm{El}$ & 0,965 \\
\hline \multirow{4}{*}{ Fabaceae } & Delonix regia (BojerexHook) Rafin & 36 & $E$ & 6,950 \\
\hline & Erythrina indica var.picta B. \& M. & 15 & $\mathrm{E}$ & 2,582 \\
\hline & Lonchocarpus campestris Mart. ex Benth. & 1 & $\mathrm{~N}-\mathrm{PR}$ & 0,193 \\
\hline & Princianella pluviosa DC. & 51 & $\mathrm{~N}$ & 9,846 \\
\hline Malvaceae & Bombax malabaricum DC. & 2 & $E$ & 0,386 \\
\hline Melastomataceae & Tibouchina granulosa (Desr.) Cogn. & 1 & $\mathrm{~N}$ & 0,193 \\
\hline Meliaceae & Melia azedarach L. & 6 & $\mathrm{El}$ & 1,158 \\
\hline \multirow{2}{*}{ Moraceae } & Ficus benjamina L. & 66 & $E$ & 12,741 \\
\hline & Ficus carica L. & 1 & $E$ & 0,193 \\
\hline \multirow{4}{*}{ Myrtaceae } & Eugenia involucrata DC & 1 & $\mathrm{~N}$ & 0,193 \\
\hline & Psidium guajava L. & 4 & $\mathrm{El}$ & 0,772 \\
\hline & Psidium sp. & 1 & N-PR & 0,193 \\
\hline & Syzygium cummini (L.) Skeels & 3 & $\mathrm{EI}$ & 0,579 \\
\hline Oleaceae & Ligustrum lucidum W T. Aiton & 219 & $\mathrm{El}$ & 42,278 \\
\hline \multirow{2}{*}{ Rosaceae } & Eriobotrya japonica (Thumb) Lindl. & 2 & El & 0,386 \\
\hline & Prunus persica (L.) Batsch & 1 & $E$ & 0,193 \\
\hline \multirow{2}{*}{ Rutaceae } & Citrus limon (L.) Burm.f. & 5 & $E$ & 0,965 \\
\hline & Murraya paniculata(L.) Jacq. & 17 & $E$ & 3,282 \\
\hline Total & & 518 & & \\
\hline
\end{tabular}

Espécies exóticas podem ocasionar graves problemas ambientais, uma vez que eventualmente realizam a competição com nativas e acarretam no desequilíbrio nos ecossistemas (GONG; CHEN; YU, 2013). A espécie L. lucidum, pode ser considerada uma ameaça constante para o paisagismo local e também para os fragmentos florestais remanescentes devido a dispersão de suas sementes, haja vista que esta e seu crescimento 
ocorrem de maneira muito rápida, impedindo a regeneração de plantas nativas. (BIONDI; MULLER, 2013). Delespinasse (2011) salienta que a espécie é a mais frequente na arborização urbana em estudo realizado em 27 munícipios paranaenses com mais de 60 mil habitantes.

No levantamento das epífitas existentes na arborização urbana de Paraná do Oeste, foram encontradas 16 espécies (Tabela 2) distribuídas em 6 famílias, com predominância das famílias Bromeliaceae, Orchidaceae e Polypodiaceae. Deve-se levar em consideração que as espécies Dendrobium nobile Lindl. Epidendrum sp, Pleurothallis sp, Syngonium podophyllum (Schott.) H.W são epífitas cultivadas, ou seja, não tiveram ocorrência natural no forófito.

Tabela 2. Levantamento florístico das epífitas encontradas no Distrito de Paraná do Oeste, munícipio de Moreira Sales - PR

Table 2. Floristic survey of the epiphytes found in the District of Paraná do Oeste, municipality of Moreira Sales - PR

\begin{tabular}{|c|c|}
\hline Família & Espécie \\
\hline Apocynaceae & Catharanthus roseus (L.) Don \\
\hline Araceae & Syngonium podophyllum Schott. \\
\hline Bromeliaceae & $\begin{array}{l}\text { Tillandsia Iolliacea Mart. ex Schult. \& Schult.f. } \\
\text { Tillandsia recurvata (L.) L. } \\
\text { Tillandsia recurvifolia Hook. } \\
\text { Tillandsia tricholepis Baker }\end{array}$ \\
\hline Cactaceae & $\begin{array}{l}\text { Epiphyllum phyllanthus (L) Haw. } \\
\text { Lepismium cruciforme (Vell.) Miq. } \\
\text { Lepismium warmingianum (K.Schum.) Barthlott }\end{array}$ \\
\hline Orchidaceae & $\begin{array}{l}\text { Acianthera sp. } \\
\text { Dendrobium nobile Lindl. } \\
\text { Epidendrum sp. } \\
\text { Pleurothallis sp. }\end{array}$ \\
\hline Polypodiaceae & $\begin{array}{l}\text { Microgramma squamulosa (Kaulf.) de la Sota } \\
\text { Pleopeltis pleopeltifolia (Raddi) Alston. } \\
\text { Pleopeltis squalida (Vell.) de la Sota }\end{array}$ \\
\hline
\end{tabular}

A abundância da família Bromeliaceae, aliada ao gênero Tilandsia já foram encontradas em outros trabalhos realizados em zonas urbanas, tais como Becker et al., (2015) em áreas urbanas da Bacia Hidrográfica do rio dos sinos - RS e Devens et al., (2016) para Luiziana - PR, com 52\% e 40\% respectivamente. De acordo com Ritter et al., (2014) este fato se deve pela facilidade de colonização destas epífitas, fazendo com que a mesma seja comum na arborização urbana das cidades.

Adicionalmente, a partir da maior frequência da espécie L. Lucidum (42,2\%) como exótica e perenófila e a constatação de exacerbada atividade de epifitísmo da espécie nativa e decídua Handroantus heptaphyllus, optou-se por analisar a interação dos indivíduos destas duas espécies com as atividades de epifitismo.

Com a análise das epífitas encontradas nas espécies Handroantus heptaphyllus, e Ligustrum Lucidum, foram encontradas trinta e oito amostras de epífitas em $H$. heptaphyllus 
pertencentes a seis espécies enquanto que em L. lucidum, obteve-se 127 epífitas, pertencentes a oito espécies (Tabela 3).

Tabela 3. H. Heptaphyllus (H.H), L. Lucidum (L.L), Abundância relativa (AR); Frequência relativa (FR); Valor indicador (IndVal) e abundância de amostras de cada espécie de epífita encontradas nos forófitos no Distrito de Paraná do Oeste, Moreira Sales, Paraná

Table 3. H. Heptaphyllus (H.H), L. Lucidum (L.L), Relative abundance (AR); Relative frequency (RF); Indicator value (IndVal) and abundance of samples of each species of epiphyte found in forophytes in the District of Paraná do Oeste, Moreira Sales, Paraná

\begin{tabular}{|c|c|c|c|c|c|c|c|c|}
\hline \multirow{2}{*}{ Epífitas } & \multicolumn{2}{|c|}{ Forófitos } & \multicolumn{2}{|c|}{$\operatorname{Ar}(\%)$} & \multicolumn{2}{|c|}{$\operatorname{Fr}(\%)$} & \multicolumn{2}{|c|}{ IndVal } \\
\hline & H.H & $L . L$ & H.H & L.L & H.H & L.L & H.H & L.L \\
\hline Dendrobium nobile Lindl. & 5 & 2 & 76 & 24 & 50 & 40 & 38 & 10 \\
\hline Lepismium cruciforme (Vell.) Miq. & 0 & 1 & 0 & 100 & 0 & 20 & 0 & 20 \\
\hline Lepismium warmingianum (K.Schum.) Barthlott & 0 & 1 & 0 & 100 & 0 & 20 & 0 & 20 \\
\hline Pleopeltis pleopeltifolia (Raddi) Alston. & 0 & 27 & 0 & 100 & 0 & 80 & 0 & 80 \\
\hline Pleopeltis squalida (Vell.) de la Sota & 0 & 4 & 0 & 100 & 0 & 40 & 0 & 40 \\
\hline Tillandsia Iolliacea Mart. ex Schult. \& Schult.f. & 20 & 31 & 45 & 55 & 50 & 80 & 22 & 44 \\
\hline Tillandsia recurvata (L.) L. & 6 & 41 & 15 & 85 & 25 & 40 & 4 & 34 \\
\hline Tillandsia recurvifolia Hook. & 1 & 0 & 100 & 0 & 25 & 0 & 25 & 0 \\
\hline Tillandsia tricholepis Baker & 3 & 20 & 16 & 84 & 25 & 40 & 4 & 34 \\
\hline
\end{tabular}

Deve-se notar que a espécie exótica invasora $L$. lucidum apresentou um número maior de epífitas que $H$. heptaphyllus. Além deste fato, as espécies $P$. squalida, $L$. cruciforme, $P$. pleopeltifolia e L. warmingianum, foram encontradas exclusivamente em L. lucidum.

A abundância de epifitismo em L. lucidum já foi constatada em outros estudos, tais como Devens et al., (2016) para a cidade de Luiziana, Ritter et al, (2014) em Farol - PR, sendo que nestes, houve um destaque para as famílias Bromeliaceae e Polypodiaceae, que também foram as famílias com maior ocorrência em Paraná do Oeste - PR.

Já a espécie $H$. heptaphyllus apresenta na área de estudo uma abundância de epifitismo, sendo que nos dez indivíduos contabilizados, foram encontradas quatro espécies de bromélias, destacando-se Tillandsia Iolliacea e uma espécie de orquídea. Este dado é corroborado por Becker et. al., (2010) que em seu estudo que englobou as áreas urbanas da Bacia Hidrográfica do Rio dos Sinos - RS, obteve $H$. heptaphyllus como o principal forófito da família Bromeliaceae.

Ao se analisar a similaridade, por meio do método de Bray-Curtis (Figura 2) evidenciase que existe uma considerável similaridade entre os indivíduos de Ligustrum lucidum, quando ás epífitas coletadas sobre eles. As amostras de Ligustrum lucidum 5 e 1 apresentam $60 \%$ de similaridade com as epífitas neles encontradas, sendo um dado significativo. De acordo com Bonnet (2007b), as árvores mais próximas à cursos d'agua ou/e remanescentes florestais 
geralmente possuem uma maior abundância ou diversidade de epífitas, esse fator é ocasionado em virtude de uma maior umidade relativa dessa porção da paisagem, originada da constante evaporação da água. Os indivíduos de Ligustrum lucidum 1 e 5 estão próximos (cerca de 50 metros) de remanescentes florestais, o mesmo acontece para as amostras de Handroantus heptaphyllus 1 e 5.

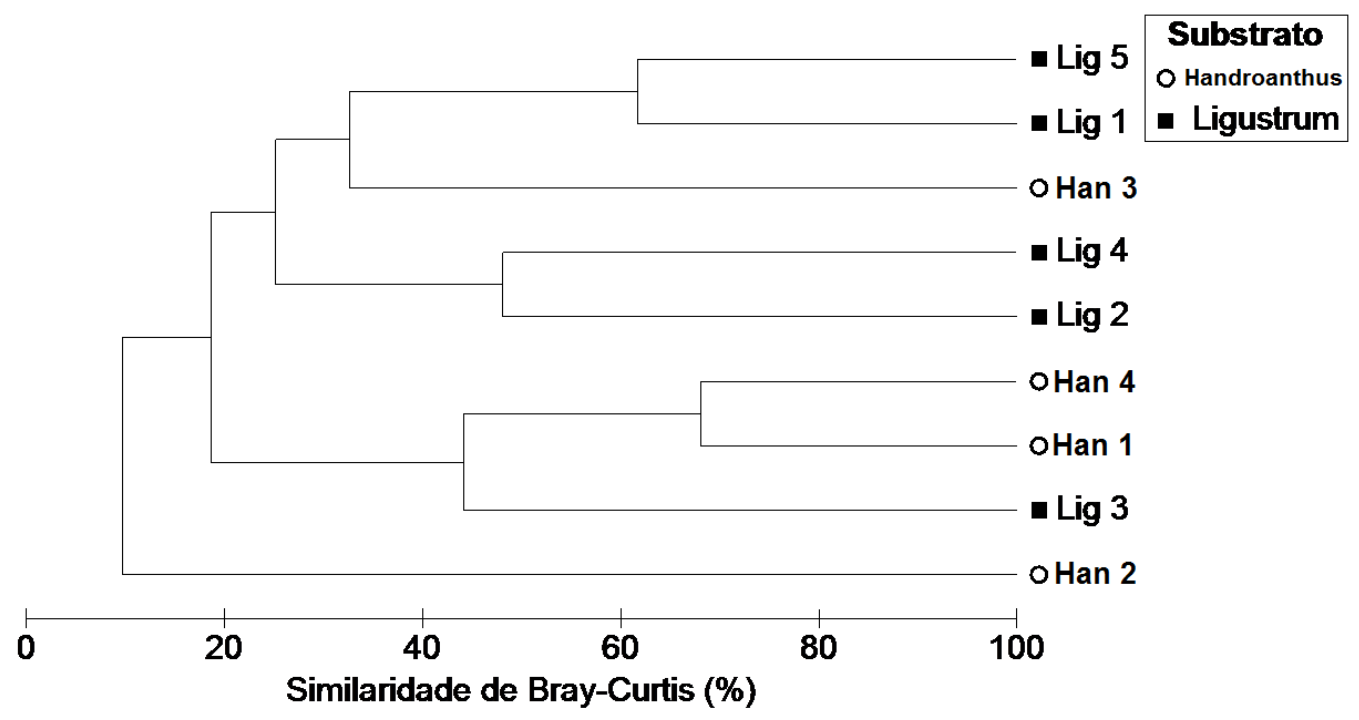

Figura 2. Gráfico de Similaridade de Bray-Curtis entre os substratos de L. Lucidum e H Heptaphylus Figure 2. Similarity Graph of Bray-Curtis between the substrates of L. Lucidum and H Heptaphylus

A umidade que vem dos remanescentes florestais é de fundamental importância para as epífitas, uma vez que as mesmas não possuem contato com o solo e dependem das partículas existentes na atmosfera para a sua sobrevivência. Nota-se então a importância da conservação dos mesmos, pois sem eles as epífitas encontrarão significativas dificuldades de se firmarem no ambiente urbano ocasionando perda de biodiversidade para o ecossistema (BONNET, 2007a).

As espécies com maior abundância relativa (Tabela 3 ) encontradas em $\mathrm{H}$. heptaphyllus foram $T$. recurvifolia e $D$. nobile, sendo a primeira exclusiva de $H$. heptaphyllus. Em $L$. Iucidum foi encontrada uma abundância maior de $P$. squalida, L. cruciforme, P. pleopeltifolia, $L$. warmingianum, sendo todas exclusivas. Algumas epífitas presentes nas duas espécies de forófitos tiveram maior frequência relativa em Ligustrum lucidum, como é o caso da $T$. loliacea (Mart.).

A espécie Pleopeltis pleopeltifolia apresentou um alto valor indicador (80) em relação a Ligustrum lucidum, sendo um indicativo de uma possível relação direta entre epífita e forófito encontrada no presente estudo. Contudo, outro fator que pode ter contribuído para esta relação é que a epífita está associada a ambientes com baixa umidade relativa, situação encontrada em determinadas épocas do ano em um ambiente urbano. A mesma é endêmica do Brasil, pertencendo à área fitogeográfica da Mata Atlântica (PRADO; HIRAI; SCHWARTSBURD, 2010). 
Pleopeltis pleopeltifolia é uma samambaia da família Polypodiaceae, sendo que o gênero Pleopeltis é caracterizados por serem epífitas e ocasionalmente rupícolas. Elas apresentam caule longo reptante, delgado, geralmente ramificado, com escamas lanceoladas, peltadas a pseudopeltadas (PRADO; HIRAI; SCHWARTSBURD, 2010). Essas escamas peltadas e pseudopeltadas se mostraram bem fixas aos galhos e ao tronco de Ligustrum lucidum, apesar de sua menor rugosidade em relação ao tronco de $H$. heptaphyllus, explicando assim a sua grande frequência relativa (Tabela 4) no forófito.

Em estudo realizado por Voytena et al (2014) a espécie Pleopeltis pleopeltifolia apresenta características anatômicas de hábito epifítico fundamentais para a sobrevivência em com períodos secos, sendo mecanismos chaves permitindo a tolerância à dessecação e reidratação da mesma. Essa resistência ao dessecamento, comum a espécies epífitas, implica no enrolamento da epífita proporcionando-a aspectos de planta seca. Esse fato possibilita a espécies do gênero Pleopeltis uma maior capacidade de sobrevivência na arborização urbana, visto que a mesma tem que se adaptar a um ambiente com condições bem diferentes aos oferecidos em locais de sua incidência natural.

Por um lado, discutem-se os malefícios e a perda de biodiversidade que a grande incidência de L. lucidum, que é espécie exótica invasora no Paraná, causa à arborização urbana (BIONDI; MULLER, 2013; OZTURK, 2008), por outro, o forófito faz interações com Pleopeltis pleopeltifolia favorecendo o epifitismo que segundo BONNET (2007b) é de fundamental importância para a preservação da biodiversidade. Handroantus heptaphyllus apesar de ser uma espécie nativa, não apresentou nenhum tipo de relação considerável com todas as espécies de epífitas coletadas.

Parece haver forte relação entre $P$. pleopeltifolia com $L$. lucidum, relacionada a pelo menos dois fatores: a maior umidade de microclima proporcionada pela copa sempre verde e pela casca mais grossa deste forófito, aliada ao acumulo de matéria orgânica nas ramificações basais. Neste sentido, recomenda-se maiores estudos que comprovem ou refutem esta hipótese.

\section{CONCLUSÕES}

No estudo das epífitas encontradas nas espécies de L. Lucidum e H. Heptaphyllus, demonstrou-se que ambas apresentam alta diversidade de indivíduos, tendo destaque as famílias Bromeliaceae, Orchidaceae e Polypodiaceae, ambas associadas a ambientes antropizados e abertos. 
Houve também a detecção de uma possível relação entre Pleopeltis pleopeltifolia com o forófito Ligustrum lucidum, podendo estar associado a fatores como matéria orgânica em suas ramificações e sua casca mais grossa, aliado a copa perene.

Porém é recomendado que sejam realizados maiores estudos para justificar essa relação, principalmente levando em consideração os fatores climáticos, que podem influenciar na abundância desta espécie.

\section{REFERÊNCIAS}

BATAGHIN, F. A.; BARROS, F.; PIRES, J. S. R. Distribuição da comunidade de epífitas vasculares em sítios sob diferentes graus de perturbação na Floresta Nacional de Ipanema, São Paulo, Brasil. Revista Brasileira de Botânica, São Paulo, v. 33, n. 3, p. 531-542, 2010.

BeRmudeZ, G. M. A.; ROdRigueZ, J. H.; PIGNATA, M. L. Comparison of the air pollution biomonitoring ability of three Tillandsia species and the lichen Ramalina celastri in Argentina. Environmental Research, v.109, p.6-14. 2009

BIONDI, D.; MULLER, E. Espécies Arbóreas Invasoras no Paisagismo dos Parques Urbanos de Curitiba, PR. Revista Floresta, Curitiba, v. 43, n. 1, p. 69-82, 2013.

BONNET, A. Conservação da biodiversidade - Em que locais se concentram as epífitas, como bromélias e orquídeas? Revista do Meio Ambiente, Niterói, v. 3, n 9, p. 31, 2007a.

BONNET, A. Epífitas: plantas que vivem sobre outras plantas. Revista do Meio Ambiente, Niterói, v. 2, n 7, p. 15, 2007b.

CERVI, A. C.; BORGO, M. Epífitos vasculares no Parque Nacional do Iguaçu, Paraná (Brasil). LEVANTAMENTO PRELIMINAR. Fontqueira, Madri, v 55, n 51, p 415 - 422, 2007.

DELESPINASSE, C. F. B.; HASSE, I.; SILVA, L. M.; CAMPESTRINI, F. Cenário da arborização urbana nas maiores cidades do estado do Paraná. Revista da Sociedade Brasileira de Arborização Urbana, Piracicaba, v. 6, n. 3, p. 149-171, 2011.

DETTKE, G. A.; ORFRINI, A. C.; MILANEZE-GUTIERRE, M. A. Composição florística e distribuição de epífitas vasculares em um remanescente alterado de Floresta Estacional Semidecidual no Paraná, Brasil. Rodriguésia, Rio de Janeiro, v. 59, n. 4, p. 859-872, 2008.

DEVENS, K. U.; GERALDINI, A. P.; AMADEO, R. M.; CAXAMBU, M. G.; MAGNONI, P. H. J. Levantamento de epífitas na arborização urbana do município de Luiziana-Paraná. Revista da Sociedade Brasileira de Arborização Urbana, Piracicaba, v. 10, n. 4, p. 1-11, 2016.

DUFENE, M.; LEGENDRE P. Species Assemblages and Indicator Species: The Need for a Flexible Asymmetrical Approach. Ecological Monographs, Washington, v. 67, n. 3, p.345-366, 1997.

EMBRAPA - Empresa Brasileira de Pesquisa Agropecuária. Levantamento de reconhecimento dos solos do estado do Paraná. Escala: 1: 250.000, 2007.

GANDOLFI, I.; CANEDOLI, C.; IMPERATO, V.; TAGLIAFERRI, I.; GKOREZIS, P.; VANGRONSVELD, J.; PADOA-SCHIOPPA, E.; PAPACCHINI, M.; FRANZETTI, A. Diversity and 
hydrocarbon-degrading potential of epiphytic microbial communities on Platanus $x$ acerifolia leaves in an urban area. Environmental Pollution, Barcelona, v. 220, p.650-658, 2017.

GERALDINO, H. C. L.; CAXAMBÚ, M. G; SOUZA, D. C. Composição florística e estrutura da comunidade de epífitas vasculares em uma área de ecótono em Campo Mourão, PR, Brasil. Acta Botanica Brasilica, Brasília, v. 24, n. 2, p. 469-482, 2010.

GONG, C ; CHEN, J; YU, S. Biotic homogenization and differentiation of the flora in artificial and near-natural habitats across urban green spaces. Landscape And Urban Planning, Amsterdam, v. 120, p.158-169, 2013.

IBGE - INSTITUTO BRASILEIRO DE GEOGRAFIA E ESTATÍSTICA. IBGE/CIDADES, MOREIRA SALES. 2016. Disponível em; < http://cidades.ibge.gov.br/xtras/perfil.php?lang $=\&$ codmun $=411610 \&$ search $=||$ infogr\%E1ficos:-informa\%E7\%F5es-completas $>$ Acesso em: 22 fev. 2016.

ITCG - Instituto de Terras, Cartografia e Geociência. Bacias Hidrográficas - Estado do Paraná. 2010. Disponível em: <http://www.geo.pr.gov.br/ms4/itcg/geo.html> Acesso em: 25 mar. 2013.

IAPAR - INSTITUTO AGRONÔMICO DO PARANÁ. Cartas Climáticas do Paraná. Londrina, Paraná, 2009. Disponível em: Acesso em http://www.iapar.br/modules/conteudo/conteudo. php?conteudo=594: 11 set. 2017.

LAPO, C. A.; MAGENTA, M. A. G. Aspectos Ecológicos da Arborização, Epifitismo e Avifauna em bairros centrais de Santos-SP. Unisanta BioScience, Santos, v. 3, n. 4, p. 226-236, 2014.

OLDFIELD, E E.; FELSON, A. J.; WOOD, S. A.; HALLETT, R. A.; STRICKLAND, M. S.; BRADFORD, M. A. Positive effects of afforestation efforts on the health of urban soils. Forest Ecology and Management, Colorado, v. 313, n. 1, p.266-273, 2014.

OZTURK, M; UYSAL, I.; GÜCEL, S.; MERT, T.; AKCICEK, E.; CELIK, S. Ethnoecology of poisonous plants of Turkey and Northern Cyprus. Pakistan Journal of Botany, Karachi, v. 4, n. 40, p.1359-1386, 2008.

PRADO, J.; HIRAI, R. Y.; SCHWARTSBURD, P. B. Criptógamos do Parque Estadual das Fontes do Ipiranga, São Paulo, SP: pteridophyta. Hoehnea, São Paulo, v. 37, n. 3, p.445-460, 2010

RITTER, C. M.; SANTOS, F. R.; CRESPAO, L. M. P.; ARDENGUI, T. C.; CAXAMBÚ, M. G. Levantamento de epífitas presentes na arborização urbana no município de Farol-Paraná. Revista da Sociedade Brasileira de Arborização Urbana, Curitiba, v. 9, n. 3, p. 18-28, 2014.

RODERJAN, C. V., GALVÃO, F., KUNIYOSHI, S. Y., HATSCHBACH, G.G. As unidades fitogeográficas do estado do Paraná, Brasil. Ciência \& Ambiente, Santa Maria, v.24, n. 1, p. 7592, 2002

ROHLF, F. J. Numerical taxonomy and multivariate analysis system. New York: 2001.

VOYTENA, A. P. L.; MINARDI, B. D.; BARUFI, J. B.; SANTOS, M.; RANDI, A. M. Pleopeltis pleopeltifolia (Polypodiopsida, Polypodiaceae), a poikilochlorophyllous desiccation-tolerant fern: anatomical,biochemical and physiological responses during water stress Australian Journal of Botany, Melbourne, v. 62, n. 1, p. 647-656, 2014. 